\title{
The influence of different reaction parameters on plant-mediated synthesis of silver nanoparticles from Citrus grandis.
}

\author{
${ }^{1}$ Nur Syazana Jalani, ${ }^{1}$ Wong Michell, ${ }^{1}$ Wong Ee Lin, ${ }^{1}$ Sharifah Zati-Hanani, ${ }^{2}$ Uda Hasim and ${ }^{1}$ Rozaini Abdullah \\ ${ }^{1}$ Department of Chemical Engineering Technology, Faculty of Engineering Technology, Universiti Malaysia Perlis, Kampus UniCITI Alam , 02100 Sungai \\ Chuchuh, Padang Besar, Perlis, Malaysia. \\ 2Institute Nano Electronic Engineering, Universiti Malaysia Perlis, 01000 Kangar Perlis, Malaysia.
}

Correspondence Author: Nur Syazana Jalani, Department of Chemical Engineering Technology, Faculty of Engineering Technology, Universiti Malaysia Perlis, Kampus UniCITI Alam , 02100 Sungai Chuchuh, Padang Besar, Perlis, Malaysia.

Received date: 12 January 2018, Accepted date: 10 March 2018, Online date: 2 April 2018

Copyright: (c) 2018 Nur Syazana Jalani et al. This is an open-access article distributed under the terms of the Creative Commons Attribution License, which permits unrestricted use, distribution, and reproduction in any medium, provided the original author and source are credited.

\begin{abstract}
A plant-mediated approach using Citrus grandis or pomelo peel extract was studied for the synthesis of silver nanoparticles (AgNPs). The effects of parameters such as $\mathrm{pH}$, incubation temperature and reaction time on the synthesis of AgNPs were also studied. It was found that the formation of AgNPs enhanced with time at higher temperature and alkaline $\mathrm{pH}$. The formation of synthesized AgNPs were confirmed by surface plasmon resonance (SPR) as determined by UV-Vis spectra around 400.5 to $414.0 \mathrm{~nm}$. Fourier transform infrared spectra (FTIR) of synthesized AgNPs showed the carboxyl, hydroxyl and amide groups on the surface of pomelo peel extract responsible for the reduction of silver ions. Size and shape of nanoparticles was analyzed by Field emission scanning microscopy-Energy Dispersive X-Ray (FESEM-EDX) shows spherical shape of nanoparticles with size ranging from 30 to $60 \mathrm{~nm}$.
\end{abstract}

Key words: Citrus grandis Silver nanoparticles Keyword 3 (At least 3 and at most 8 keywords)

\section{INTRODUCTION}

Silver nanoparticles (AgNPs) have gained special focus as they are favorable in various fields including electronic devices [1], chemical or biological sensing [2] and pharmaceuticals [3].

Previously, synthetic reducing agents such as ethylene glycol, sodium borohydrate and formaldehyde are used to synthesize AgNPs which are being viewed as traditional chemical method [4]. The usage of chemicals in synthesis can be poisonous thus threatening the environment and human health. Moreover, the procedures maybe tedious and economically expensive to be carried out at an industrial scale. Therefore, it is necessary to search for inexpensive, trustworthy and safer approach to the synthesis of stable metal nanoparticles.

Use of plant extract for the synthesis of metal nanoparticles have been suggested as possible ecofriendly alternatives to chemical and physical methods. Several extensive literature review revealed that reports on the successful synthesis of AgNPs using plant materials with different part such as fruit [5], bark [6], fruit peels [7], root [8] and callus [9]. The plant extract has been widely investigated due to their inexpensive and simple procedure [10].

In this study, Citrus Grandis or pomelo peel extract was utilized for the synthesis of AgNPs. There are several research works on synthesizing AgNPs using Citrus species such as Citrus sinesis [11], Citrus limon [12], Citrus reticulate [13] and Citrus aurantium L. [14]. The broad range of biomolecules in Citrus fruit such as alkaloids, terpenoids, phenols, flavanoids, tannins, quinines are known to facilitate the synthesis of metal nanoparticles by reducing $\mathrm{Ag}^{+}$to $\mathrm{Ag}^{0}$ ions and organized the $\mathrm{Ag}^{0}$ into AgNPs.

\section{MATERIALS AND METHODS}

2.1 Materials:

2.2 Preparation of pomelo peel extract:

Citrus Grandis fruits were obtained from the local market. The fresh peels of Citrus Grandis were dried completely in oven for 18 hours. The dried fruit peels were incised into small pieces and powdered into fine particles. About $10 \mathrm{~g}$ of the powdered fruit peel was mixed with $500 \mathrm{~mL}$ distilled water and then incubated in water bath for 30 minutes at $60^{\circ} \mathrm{C}$. Then, peel extract was filtered through Whatman No.1 filter paper and the resulting filtrate was stored at $4^{\circ} \mathrm{C}$ for further use.

2.3 Biosynthesis of silver nanoparticles:

2.3.1 Effect of $\mathrm{pH}$ :

Effect of initial $\mathrm{pH}$ on AgNPs synthesis was investigated over a wider $\mathrm{pH}$ range $(\mathrm{pH} 3.0,7.0,9.0$ and 11.0). The $\mathrm{pH}$ of the reaction mixtures were adjusted using hydrochloric acid or sodium hydroxide. The other reaction conditions which included $5 \mathrm{~mL}$ of peel extract in $50 \mathrm{~mL}^{\circ} \mathrm{AgNO}_{3}$ solution, incubation temperature of $60^{\circ} \mathrm{C}$ and reaction time of 15 minutes were kept constant. 
The effect of incubation temperature on AgNPs synthesis was studied by varying the incubation temperature of the reaction mixtures to $25,40,60$ and $80^{\circ} \mathrm{C}$. Reaction time of 15 minutes and $\mathrm{pH} 7.0$ were kept constant for $5 \mathrm{~mL}$ of peel extract in $50 \mathrm{~mL}$ of $1 \mathrm{mM} \mathrm{AgNO} 3$ solution throughout the change of incubation temperature.

\subsubsection{Effect of reaction time:}

The influence of reaction time on synthesis of AgNPs was carried out from 15 to 90 minutes. Other reaction conditions were kept constant at incubation temperature of $60^{\circ} \mathrm{C}$ and $\mathrm{pH} 7.0$ for $5 \mathrm{~mL}$ of peel extract in $50 \mathrm{~mL}$ of $1 \mathrm{mM}$ silver nitrate solution.

\subsection{Characterization of synthesized silver nanoparticles:}

The optical absorption spectra of the synthesized AgNPs were observed by using UV-1800 Shimadzu spectrophotometer. FTIR analysis was carried out after centrifugation of residual solution at $13000 \mathrm{rpm}$ for 15 minutes and then the suspension was dispersed in $1 \mathrm{~mL}$ of sterilized distilled water. This was to ensure complete removal of biomolecules that were not absorbed by the nanoparticles. The morphology and size of freeze dried AgNPs were evaluated using FeSEMEDX (Model FEI Nova NanoSEM 450).

\section{RESULT AND DISCUSSION}

\subsection{Visual observation:}

The early prediction of AgNPs formation is primarily monitored by colour change. The color changes from faint yellow to brownish colour as shown in Figure 1.

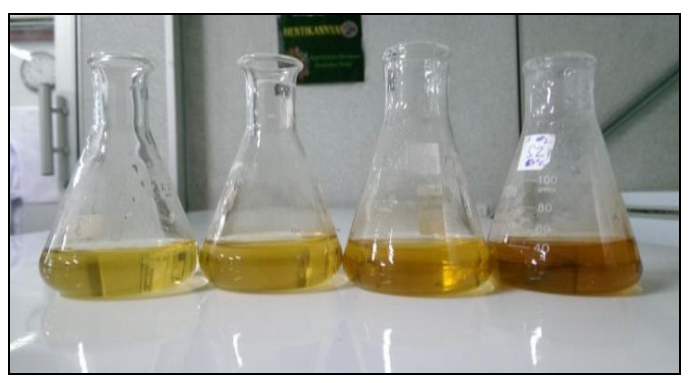

Fig. 1: Colour changes of colloidal solution of AgNPs

This indicating the generation of silver nanoparticles due to the reduction of silver metal ions $\mathrm{Ag}^{+}$into silver nanoparticles $\left(\mathrm{Ag}^{\circ}\right)$ which is attribute from the active compounds present in pomelo peel extract. Different colour changes in synthesis of silver nanoparticles are as a result of light absorption and scattering in the visible region based on surface plasmon resonance (SPR) [15].

SPR is a combination of collective oscillation of conduction electrons with the incident light; in other words, they are fundamentally the light waves that are trapped on metal surface and interacted with the free electrons of silver [16]. The excitation is also caused by an inhomogeneous polarization of the nanoparticles by the electromagnetic field as the particle size becomes comparable to the wavelength of the incoming radiation [17].

\subsection{UV-Vis spectral analysis:}

UV-Vis spectroscopy is a spectral technique used to confirm the formation of AgNPs in colloidal solution. The SPR absorption band obtained for different parameters are within the range of 390-430 nm which is prescribed for AgNPs [18]. Hence the results obtained ensure the existence of AgNPs in the colloidal solution.

The optical properties observed in UV-visible spectra of AgNPs result in the peak broadening or shifts of the absorption wavelength. When the rate of nanoparticles biosynthesis increases, the plasmon absorption maximum shifts to longer wavelengths as the bandwidth increases. In addition, the broadening of band in UV-Vis spectra indicated that the particles are polydispersed [19]. The observed SPR wavelength also depends on the size of AgNPs. This is caused by larger sizes of nanoparticles exhibit increased scattering and result in broaden band that shift towards longer wavelengths [20]. Besides that, an increase in the intensity of SPR absorption band signifying an enhancement in the formation of AgNPs [21].

\subsubsection{Effect of $\mathrm{pH}$ :}

Initial $\mathrm{pH}$ plays an important role in the biosynthesis of silver nanoparticles. The effect of $\mathrm{pH}$ on synthesis of silver nanoparticles was studied by varying the $\mathrm{pH}$ range as shown in Figure 2. The SPR wavelength varied from the range of 400.5 to $408.0 \mathrm{~nm}$ as the $\mathrm{pH}$ increased from $\mathrm{pH} 3$ to $\mathrm{pH} 11$.

There were no characteristic band of AgNPs observed in strong acidic condition $(\mathrm{pH} 3)$. This indicates no formation of AgNPs under acidic condition. The increasing $\mathrm{pH}$ from $\mathrm{pH} 7$ to $\mathrm{pH} 11$ results the SPR absorption band become intense and shifted towards lower wavelength which indicate smaller particle size [22]. These observations indicate that $\mathrm{pH}$ within this range can initiate the synthesis of AgNPs. Meanwhile, the UV-Visible absorption spectra at $\mathrm{pH} 11$ has the maximum absorption which is attributed to the band of SPR wavelength at $400.50 \mathrm{~nm}$. Thus, the optimum pH can be concluded at pH 11.

Higher intensity of SPR absorption band at alkaline condition specifies increasing in number of smaller silver nanoparticles [23]. These observation supports the size of nanoparticles observed in FESEM image (Fig. 7).

The increase in $\mathrm{pH}$ to alkaline range enhances the reduction potentials of all functional groups thus allow the formation of smaller nanoparticles. Hence, alkaline condition is favorable for the biosynthesis. The possible reason would be due to the availability of more $\mathrm{H}^{+}$from the metabolites at higher $\mathrm{pH}$ which enables quicker reduction of silver nitrate and hence the oxidation of the metabolites. The stability of the nanoparticles at alkaline $\mathrm{pH}$ may be due to the optimal activity of the enzymes and capping proteins present in plant extracts [24].

Meanwhile at acidic condition, broadening SPR absorption band was observed which indicates the formation of large size of nanoparticles [25]. This statement strongly supports the nanoparticles size obtained from FESEM image (Fig. 6). 


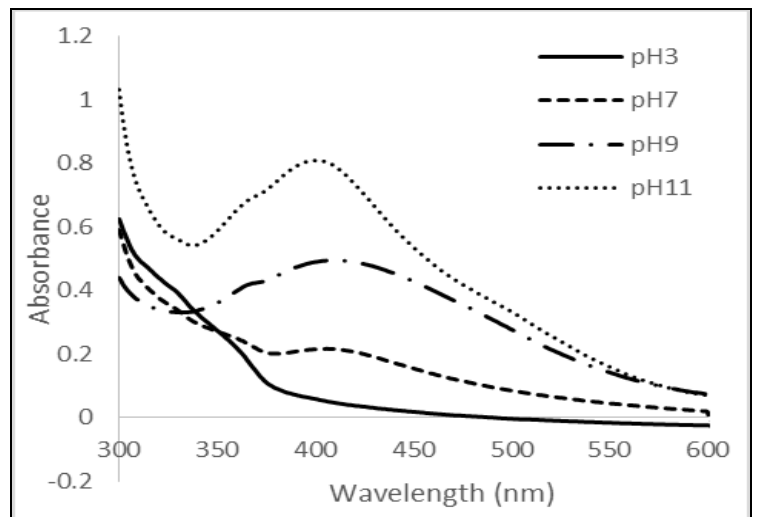

Fig. 2: UV-Vis absorption spectra of synthesized AgNPs at different $\mathrm{pH}$

The nanoparticles aggregation surpasses the nucleation process and decreases the synthesis of silver nanoparticles due to there are no indication of SPR wavelength in UV-vis spectra [26]. Higher positive charges at the surface of nanoparticles attract negative charge biomass and leads to flocculation in the reaction medium. Thus, even if the nanoparticles form, yet they are not stable enough to avoid agglomeration.

\subsubsection{Effect of incubation temperature:}

Temperature is another physical factor which plays an important role to control the nucleation process of nanoparticle formation. Figure 3 showed the reaction mixtures were incubated at temperature of $25,40,60$ and $80^{\circ} \mathrm{C}$. The SPR wavelength varied from 400.5 to $409.5 \mathrm{~nm}$.

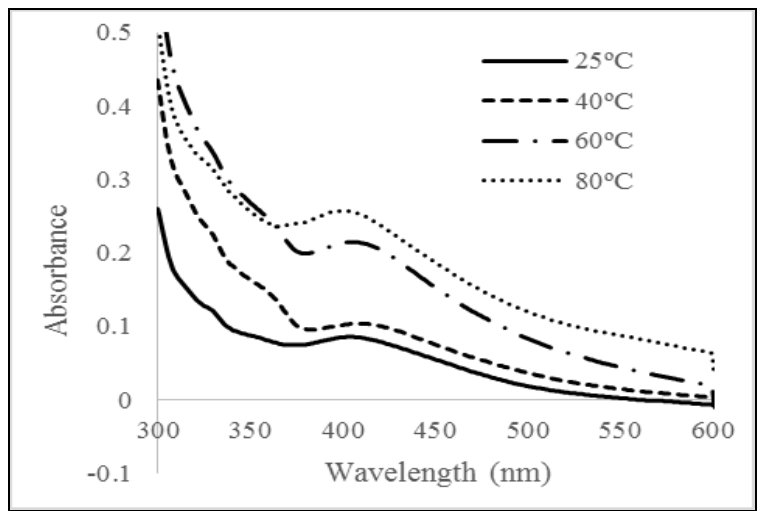

Fig. 3: UV-Vis absorption spectra of synthesized AgNPs at different incubation temperature

The reaction mixtures that incubated at $25^{\circ} \mathrm{C}$ and $40^{\circ} \mathrm{C}$ showed less pronounced SPR absorption band whereas at higher incubation temperature $\left(60^{\circ} \mathrm{C}\right.$ and $80^{\circ} \mathrm{C}$ ) more intense SPR absorption band was observed. The SPR absorption band becomes sharper with the increasing of temperature and the maximum intensity of absorption spectra was detected at $80^{\circ} \mathrm{C}$. The increase in absorbance with incubation temperature rendered the synthesis of AgNPs [27].

As the reaction temperature increases, the reduction rate increases and thus most silver ions are consumed rapidly in the formation of nuclei, retarding the secondary reduction process on the surface of the preformed nuclei [28].

\subsubsection{Effect of reaction time:}

Reaction time is another factor affecting the formation of silver nanoparticles. The reaction mixtures were incubated for 15, 30, 60 and 90 minutes. Figure 4 illustrates the increasing of reaction time resulted in gradual increase of absorbance spectrum. The SPR wavelength shifted to longer wavelength from 406 to 414 $\mathrm{nm}$ as the reaction time increased. The increasing in intensity of the SPR absorption band with reaction time indicates the increase in formation of the AgNPs.

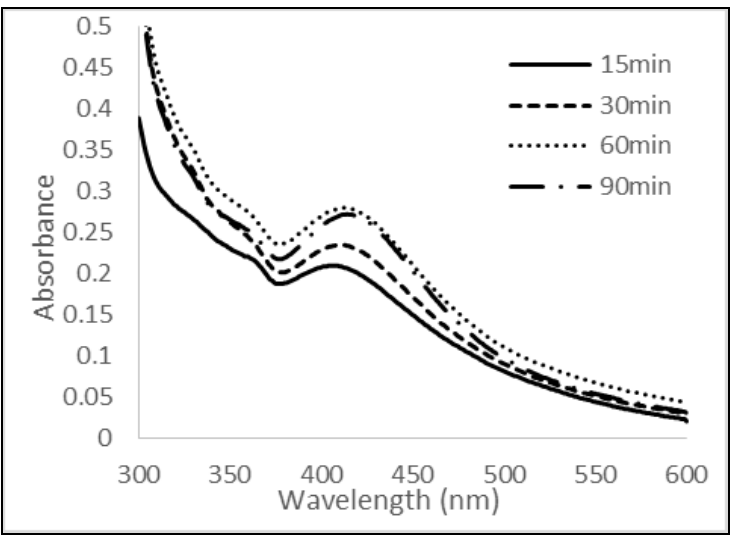

Fig. 4: UV-Vis absorption spectra of synthesized AgNPs at different reaction time 
The intensity of SPR absorption band was the highest when the reaction time reaches 60 minutes and it undergoes a reduction when the reaction time is further extended to $90 \mathrm{~min}$. Eventually, there is no change in SPR wavelength after $414 \mathrm{~nm}$ indicating a sign of completion of silver ions reduction and stability of the AgNPs colloidal solution within the reaction period [29].

\subsection{FTIR analysis:}

FTIR analysis was carried out to identify the major functional groups in Citrus Grandis peel extract and their possible involvement in AgNPs synthesis. The band intensities in different regions of the spectrum for the pomelo peel extract and synthesized silver nanoparticles were analyzed and are shown in Figure 5 below.

The shifts in the region 3700 to $3100 \mathrm{~cm}^{-1}$ and 3000 to $2850 \mathrm{~cm}^{-1}$ were assigned to $\mathrm{O}-\mathrm{H}$ stretching of alcohol, phenol compounds, and water which suggest the involvement of these compounds in AgNPs synthesis. The peaks shift in the region of 1860 to $1550 \mathrm{~cm}^{-1}$ and 1480 to $1375 \mathrm{~cm}^{-1}$ which correspond to N-H bend of primary and secondary amides and $\mathrm{C}-\mathrm{H}\left(-\mathrm{CH}^{3}-\right.$ bend $)$ of alkanes, respectively. Another shift was observed at the region of 1350 to $1000 \mathrm{~cm}^{-1}$ correspond to $\mathrm{C}-\mathrm{N}$ stretching vibration of the amine or $\mathrm{C}-\mathrm{O}$ stretching of alcohols, ethers, carboxylic acids, esters and anhydrides.

The shifting of these peaks indicated that carboxyl, hydroxyl and amide groups on the surface of pomelo peel extract may be participating in the process of nanoparticle synthesis [30].

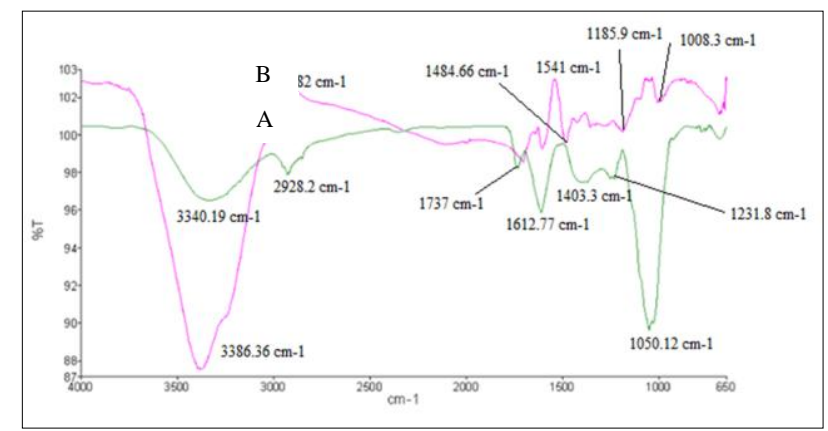

Fig. 5: FTIR spectrum of Citrus Grandis peel extract (A) before (B) after reaction with silver nitrate

\subsection{FESEM-EDX analysis:}

FESEM-EDX analysis was employed to observe the morphology of the nanoparticles synthesized under $\mathrm{pH} 3$ and $\mathrm{pH} 11$.

The shape of the particles synthesized under $\mathrm{pH} 3($ lower $\mathrm{pH})$ is less regular and more aggregate as shown in Figure 6 below.

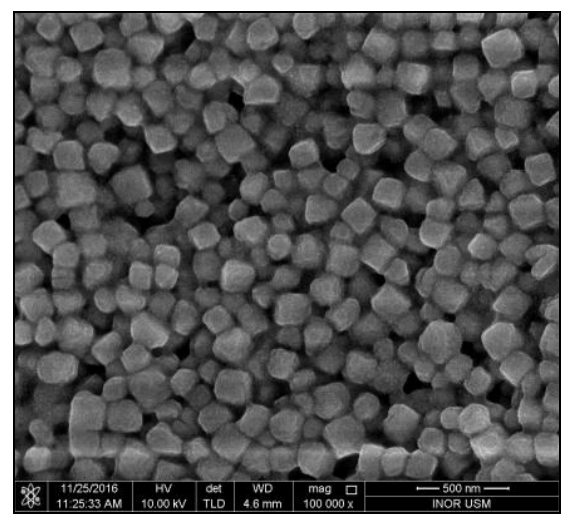

Fig. 6: FESEM image of synthesized silver particles at $\mathrm{pH} 3$

The silver particles aggregate to form larger particles rather than to nucleate and form new particles. Most of the synthesized silver particles are found to be in size range between 100-170 $\mathrm{nm}$ which is beyond nano size. This is close agreement to [31] who reported agglomeration surpassed the nucleation process in acidic condition and thus diminished the synthesis of AgNPs.

In contrast, it was observed that the nanoparticles were dispersed and spherical in shape under pH 11 (alkaline condition) in Figure 7 below. The size of synthesized AgNPs ranging from 30 to $60 \mathrm{~nm}$.

Higher $\mathrm{pH}$ facilitates the nucleation and subsequent formation of large number of nanoparticles with smaller diameter. This statement is supported by [32] which also obtained small size of nanoparticles at high $\mathrm{pH}$.

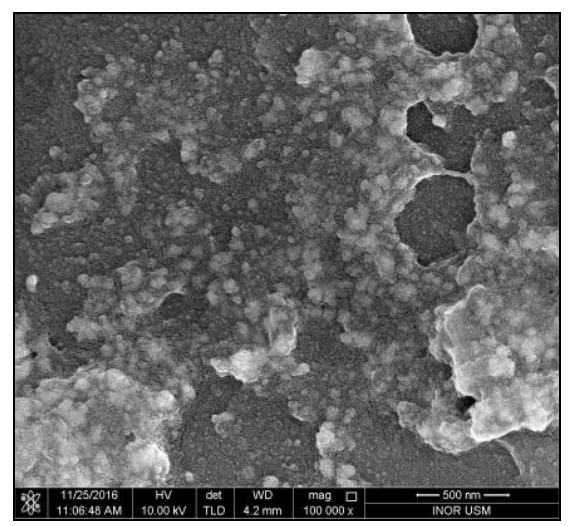



parameters on plant-mediated synthesis of silver nanoparticles from Citrus grandis. Advances in Natural and Applied Sciences., 12(4): 1-5.

The EDX analysis provides qualitative as well as quantitative measurements of elements that may be involved in formation of AgNPs. Figure 8 showed the EDX profile of AgNPs whereby the strong peak at $3 \mathrm{keV}$ indicating the presence of elemental silver which attributed by their surface plasmon resonance [33]. The EDX profile also exhibits the presence of carbon and oxygen with Ag, implying that AgNPs are capped by the organic components present in pomelo peel extract for explicating the bioconversion of the silver salt into AgNPs [34]. The others signal from $\mathrm{C}, \mathrm{O}, \mathrm{Na}$ and $\mathrm{Si}$ atoms are also recorded which might be attributed to pomelo peel extract used.

Fig. 8: EDX profile of synthesized AgNPs

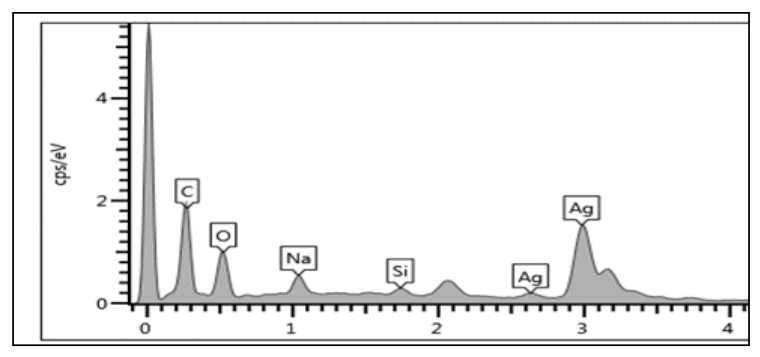

Conclusion:

AgNPs have been successfully synthesized using pomelo peel extract as natural reducing agent. The synthesis of AgNPs depends on the pH, incubation temperature and reaction time. It is confirmed that the formation of AgNPs in colloidal solution by UV-Vis analysis which exhibits the SPR absorption band at range of 400.5 to $414.0 \mathrm{~nm}$. From FESEM-EDX analysis, the synthesized silver particles tend to agglomerate and formed larger particles in acidic condition. Meanwhile, smaller AgNPs were obtained in alkaline condition with spherical shape. The FTIR analysis also revealed that the biological molecules in pomelo peel extract could possibly perform dual functions of formation and stabilization of silver nanoparticles in the aqueous medium.

It can be concluded that the synthesis of AgNPs is optimum with time at higher temperature and alkaline condition.

\section{ACKNOWLEDGEMENT}

This work has been supported by Ministry of Higher Education through Fundamental Research Grant Scheme (FRGS) under a grant number of FRGS 900300509. The authors were gratefully acknowledged Faculty of Engineering Technology and Institute of Sustainable Agrotechnology, Sungai Chuchuh for providing facilities to carry out this research. The author also appreciatively acknowledged Institute Nano Electronic Engineering for facilities and consultation support.

\section{REFERENCES}

[1] Wang, T., Kaempgen, M., Nopphawan, P., Wee, G., Mhaisalkar, S., \& Srinivasan, M. (2010). Silver nanoparticle-decorated carbon nanotubes as bifunctional gas-diffusion electrodes for zinc-air batteries. Journal of Power Sources, 195(13), 4350-4355.

[2] Ahmed, S., Ahmad, M., Swami, B. L., \& Ikram, S. (2016). A review on plants extract mediated synthesis of silver nanoparticles for antimicrobial applications: a green expertise. Journal of advanced research, 7(1), 17-28.

[3] Begum, N. A., Mondal, S., Basu, S., Laskar, R. A., \& Mandal, D. (2009). Biogenic synthesis of Au and Ag nanoparticles using aqueous solutions of Black Tea leaf extracts. Colloids and surfaces B: Biointerfaces, 71(1), 113-118.Sahoo, P., S. Kamal, T. Kumar, B. Sreedhar, A. Singh and S. Srivastava, 2009. Sci. J., 59.

[4] Prathna, T. C., Chandrasekaran, N., Raichur, A. M., \& Mukherjee, A. (2011). Kinetic evolution studies of silver nanoparticles in a bio-based green synthesis process. Colloids and Surfaces A: Physicochemical and Engineering Aspects, 377(1-3), 212-216.Iravani, S. and B. Zolfaghari, 2013. BioMed Res. Int., 2013.

[5] Rao, N.H.N.L., S.V. Pammi, P.S.G. Kollu and P.L. Mater, 2016. Sci. Eng. C. Mater. Biol. Appl., 62.

[6] Ahmad, N., Sharma, S., Alam, M. K., Singh, V. N., Shamsi, S. F., Mehta, B. R., \& Fatma, A. (2010). Rapid synthesis of silver nanoparticles using dried medicinal plant of basil. Colloids and Surfaces B: Biointerfaces, 81(1), 81-86.Nabikhan, A., K. Kandasamy, A. Raj and N.M. Alikunhi, 2010. Colloids Surf. B, 79 .

[7] Christensen, L., Vivekanandhan, S., Misra, M., \& Mohanty, A. K. (2011). Biosynthesis of silver nanoparticles using murraya koenigii (curry leaf): an investigation on the effect of broth concentration in reduction mechanism and particle size. Advanced Materials Letters, 2(6), 429-434..

[8] Kaviya, S., Santhanalakshmi, J., Viswanathan, B., Muthumary, J., \& Srinivasan, K. (2011). Biosynthesis of silver nanoparticles using Citrus sinensis peel extract and its antibacterial activity. Spectrochimica Acta Part A: Molecular and Biomolecular Spectroscopy, 79(3), 594-598..

[9] Prathna, T. C., Chandrasekaran, N., Raichur, A. M., \& Mukherjee, A. (2011). Biomimetic synthesis of silver nanoparticles by Citrus limon (lemon) aqueous extract and theoretical prediction of particle size. Colloids and Surfaces B: Biointerfaces, 82(1), 152-159.

[10] Sujitha, M. V., \& Kannan, S. (2013). Green synthesis of gold nanoparticles using Citrus fruits (Citrus limon, Citrus reticulata and Citrus sinensis) aqueous extract and its characterization. Spectrochimica Acta Part A: Molecular and Biomolecular Spectroscopy, 102, 15-23.

[11] Rajasekharreddy, P., Rani, P. U., \& Sreedhar, B. (2010). Qualitative assessment of silver and gold nanoparticle synthesis in various plants: a photobiological approach. Journal of Nanoparticle Research, 12(5), 1711-1721..

[12] Zielińska, A., Skwarek, E., Zaleska, A., Gazda, M., \& Hupka, J. (2009). Preparation of silver nanoparticles with controlled particle size. Procedia Chemistry, 1(2), 1560-1566.Kannan, N., and S. Subbalaxmi, 2011. Res. J. Nanosci. Nanotechno., 1.

[13] Ayyad, O., Muñoz-Rojas, D., Oró-Solé, J., \& Gómez-Romero, P. (2010). From silver nanoparticles to nanostructures through matrix chemistry. Journal of Nanoparticle Research, 12(1), 337-345.Tang, L., Y. Ye, H. Zhang, C. Liao, S. Zong, Y. Cui, and J. Zhang, 2014. Nano, 09.

[14] Ponarulselvam, S., Panneerselvam, C., Murugan, K., Aarthi, N., Kalimuthu, K., \& Thangamani, S. (2012). Synthesis of silver nanoparticles using leaves of Catharanthus roseus Linn. G. Don and their antiplasmodial activities. Asian Pacific journal of tropical biomedicine, 2(7), 574-580.Jha, A.K. and K. Prasad, 2016. Adv. Mat., 7.

[15] Verma, A., \& Mehata, M. S. (2016). Controllable synthesis of silver nanoparticles using Neem leaves and their antimicrobial activity. Journal of radiation Research and applied sciences, 9(1), 109-115., 9.

[16] Kumar, B., Smita, K., Debut, A., \& Cumbal, L. (2016). Extracellular green synthesis of silver nanoparticles using Amazonian fruit Araza (Eugenia stipitata McVaugh). Transactions of Nonferrous Metals Society of China, 26(9), 2363-2371.Sumi Maria, B., A. Devadiga, V. Shetty Kodialbail and M.B. Saidutta, 2015. Applied Nanoscience, 5.

[17] Jayapriya, E., \& Lalitha, P. (2013). Synthesis of silver nanoparticles using leaf aqueous extract of Ocimum basilicum (L.). International Journal of ChemTech Research, 5(6), 2985-2992.Vanaja, M., S. Rajeshkumar, K. Paulkumar, G. Gnanajobitha, C. Malarkodi, G. Annadurai, 2013. Adv. Appl. Sci. Res., 4.

[18] Sathishkumar, M., Sneha, K., Won, S. W., Cho, C. W., Kim, S., \& Yun, Y. S. (2009). Cinnamon zeylanicum bark extract and powder mediated green synthesis of nano-crystalline silver particles and its bactericidal activity. Colloids and Surfaces B: Biointerfaces, 73(2), 332-338.Ahmad, B., J. Ali and S. Bashir, 2013. World Appl. Sci. J., 22.

[19] Ahmed, S., Ahmad, M., Swami, B. L., \& Ikram, S. (2016). A review on plants extract mediated synthesis of silver nanoparticles for antimicrobial applications: a green expertise. Journal of advanced research, 7(1), 17-28.

[20] Verma, D.K., S.H. Hasan and R.M. Banik, 2015. Res. Sci. Eng. Tech., 4. 
[21] Bankar, A., Joshi, B., Kumar, A. R., \& Zinjarde, S. (2010). Banana peel extract mediated novel route for the synthesis of silver nanoparticles. Colloids and Surfaces A: Physicochemical and Engineering Aspects, 368(1-3), 58-63.

[22] Mohamad, N. A. N., Arham, N. A., Jai, J., \& Hadi, A. (2014). Plant extract as reducing agent in synthesis of metallic nanoparticles: a review. In Advanced Materials Research (Vol. 832, pp. 350-355). Trans Tech Publications..

[23] Roopan, S. M., Madhumitha, G., Rahuman, A. A., Kamaraj, C., Bharathi, A., \& Surendra, T. V. (2013). Low-cost and eco-friendly phyto-synthesis of silver nanoparticles using Cocos nucifera coir extract and its larvicidal activity. Industrial Crops and Products, 43, 631-635.

[24] Chitra, K., \& Annadurai, G. BioMed Res. Int., 2014, 1 (2014).

Manikandan R, Manikandan B, Raman T,Arunagirinathan K, Prabhu NM, Jothi Basu M, Perumal M, Palanisamy S, Munusamy A. Biosynthesis of silver nanoparticles using ethanolic petals extract of Rosa indica and characterization of its antibacterial,anticancer and anti-inflammatory activities. Spectrochim Acta A Mol Biomol Spectrosc. 2015 5; 138: 120-129. 